\title{
Study of SAW Based on a Micro Force Sensor in Wireless Sensor Network
}

\author{
Jun Wang, ${ }^{1}$ Yuanyuan Li, ${ }^{1}$ Ke Chen, ${ }^{1}$ Wenke Lu, ${ }^{2}$ Qinghong Liu, ${ }^{3}$ \\ Haoxin Zhang, ${ }^{3}$ and Huashan Yan $^{4}$ \\ ${ }^{1}$ College of Electronic and Electrical Engineering, Shanghai University of Engineering Science, Shanghai 201620, China \\ ${ }^{2}$ Electronics and Information Engineering, Donghua University, Shanghai 201620, China \\ ${ }^{3}$ Xian Leitong Science \& Technology Co., Ltd., Xian 710049, China \\ ${ }^{4}$ Shanghai Advanced Traction Battery Systems Co., Ltd., Shanghai 201805, China
}

Correspondence should be addressed to Yuanyuan Li; liyuanyuanedu@163.com

Received 24 November 2016; Revised 7 March 2017; Accepted 15 March 2017; Published 19 April 2017

Academic Editor: Lu Liu

Copyright (c) 2017 Jun Wang et al. This is an open access article distributed under the Creative Commons Attribution License, which permits unrestricted use, distribution, and reproduction in any medium, provided the original work is properly cited.

Wireless sensor network (WSN) technology has increasingly assumed an active role in detection, identification, location, and tracking applications after more than ten years of development. However, its application still suffers from technology bottlenecks, which must be solved and perfected to eliminate the key problems of the technology. This article investigates WSN acquisition nodes and analyzes the relationship between the frequency and actual pressure values of sensor nodes. The sensitive mechanism of the surface acoustic wave (SAW) based on a micro force sensor is researched, and the principle of least squares method is used to establish a transformation model of frequency and pressure for the SAW sensor. According to the model, polyfit function and matrix calculation are selected to solve and calculate the estimate of the polynomial coefficients, which simulate the data acquisition of WSN nodes and draw a polynomial curve fitting. The actual SAW sensor is tested to demonstrate the reasonableness of the device stability in WSNs.

\section{Introduction}

Wireless sensor network (WSN) technology has various applications in many disciplines and fields, such as communication, embedded computing, data processing, torrent analysis, and sensor technology. A set of integrated sensing, information processing, and communication units of wireless sensor nodes constitute WSNs without infrastructure [1]. Wireless sensor nodes in WSNs monitor and collect data in their coverage areas; they then collaborate with nodes and send the collected data to the coordinator nodes by multihop wireless through transmission channels [2]. The coordinator nodes then use the Internet or other channels to interact with users. WSNs have significant advantages in replacing traditional wired network transmission modes [3]. The important scientific and practical values of WSNs have gained much attention from many researchers and have been recognized as an emerging research field since the 2000s [4]. WSNs mix the logical information and real physical worlds together and transform the interaction between humans and nature $[5,6]$.

The monitoring environment is currently becoming increasingly complex. Simple data acquired by traditional sensor networks cannot meet the full requirements of environmental monitoring. Achieving fine-grained and accurate monitoring information is urgently needed. Actual application demands indicate that WSNs are usually composed of hundreds of sensor nodes, which are specifically or randomly deployed based on the interest within a particular geographical or physical area. These nodes sense factors in the external world, such as temperature, humidity, pressure, vibration, magnetic field, and light intensity; they also complete observations in target detection, identification, location, and tracking [7-10]. Surface acoustic wave (SAW) is an interdisciplinary subject that includes acoustics, electronics, piezoelectric materials, and semiconductor technology applied in the rapid development of large-scale integrated circuits of microelectronics, computer, silicon micromechanical processing, 
semiconductor planar process, and laser technologies. SAW sensors have excellent properties, such as passivity, small size, low cost, excellent temperature stability, high reliability, and high reproducibility, which overcome the complicated algorithms and high power of VLSI [11, 12] and the large size and low reproducibility of optical devices [13]. The development of SAW based on a micro force sensor has been oriented toward intelligent wireless networks $[14,15]$. Frequency and pressure conversion in the network optimization problem of SAW pressure sensors has become an urgent research topic. The method of implementing wavelet transform using SAW devices was first proposed by Zhu and Lu. Nowadays, micromanipulation has performed to design the precise pressure device in WSNs [16]. In order to design precise pressure devices in WSNs, micromanipulation is currently performed.

Different devices for intelligent and wireless networks have been developed and integrated. Lü established the force model whose device stability and precision of elastic modulus analysis should be improved [17]. Jungwirth described a micromechanical precision pressure sensor in delay lines [18]. Muntwyler designed a three-axis micro force sensor, which suffers from the problem of measurement uncertainty [19]. The present study constructs a SAW sensor based on a micro force sensor device on two sides and forcemeasuring elements that use a cantilever beam. The device is also equipped with single channel inductive components, and its electromagnetic field interference is more sensitive than that of others. Two inductors are placed at $90^{\circ}$ to decrease the interference between them. A mathematical model is also established to analyze the frequency of the varied pressure. The SAW based on a micro force sensor has good linearity, consistency, and repeatability in performance within the scope of the effective measurement. It also uses the piezoelectric properties and temperature stability of crystals, which enhances the signal processing of this device in terms of its digital features and stable WSN performance.

The velocity and phase of the SAW based on a micro force sensor are changed after sensing the change in the quantity, which is generated by the interdigital transducer (IDT) of the SAW device. The output signal is directly measured by the network analyzer. The rest of this paper is organized as follows: Section 1 presents the background research on WSNs. Section 2 discusses the pressure-sensitive mechanism of a SAW based on a micro force sensor. Section 3 develops a simulation model using the principle of the least squares method. Sections 1-4 shift the focus of the study to the relationship between frequency and pressure, which in turn obtains the relationship between the output signal and sensing volume. Section 5 presents the testing and analysis of the SAW based on a micro force sensor used under different pressure levels. Section 6 analyzes temperature compensation of SAW based on a micro force sensor.

\section{WSNs}

2.1. Concept of WSNs. WSNs are a multihop self-organizing network system by wireless communication, which is composed of a large number of sensor nodes in monitoring areas [20]. Its purpose is to perceive, collect, and process the information of objects in the network and send it to observers.

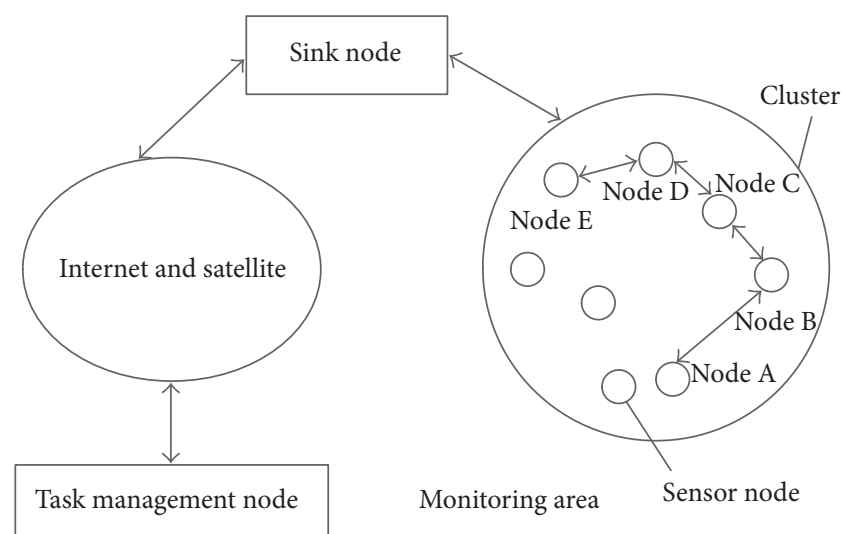

FIGURE 1: WSN architecture.

The structure of WSNs is shown in Figure 1. Sensor network system usually consists of sensor nodes, sink nodes, and management nodes.

In wireless sensor networks, sensor nodes are deployed in monitoring areas for automatic detection. Each sensor node sends out wireless signals and then constructs a network that supports dynamic topology in a self-organizing manner. In this network, the node has some association with cluster. In the cluster, usually according to certain rules, a sink node (such as nodes A, B, C, D, and E in Figure 1) is to be elected. The data is processed via the Internet or satellite system.

2.2. Wireless Sensor Network Node. Sensor node is the basic unit of wireless sensor network, which is composed of processor module, wireless communication module, sensor module, and power management module as shown in Figure 2. Sensor module is mainly composed of various types of sensors, $\mathrm{AD} / \mathrm{DC}$ conversion module structure. Due to different physical signals, different types of sensors are used for data acquisition, and then data are transmitted to the processor module for processing. And the processor module is computing core of wireless sensor network node whose design requires miniaturization, low cost, stability, and security. Data communication protocols of sensor network in wireless communication module include physical layer, link layer, network layer, and application layer. The power management module not only provides the necessary energy for operation of sensor nodes, but also provides necessary power management to prolong the lifetime of wireless sensor networks.

WSNs have a very close correlation, and different applications require different network models, hardware platforms, and system software. In the design of sensor nodes, the following aspects should be considered.

(1) Miniaturization. Wireless sensor network node requirements are as small as possible on the volume, without affecting the performance of the system.

(2) Low Cost. Low cost is the basic requirement of WSN node.

(3) Stability and Security. Under conditions of given temperature, humidity, and pressure, each module of sensor node can work normally. 


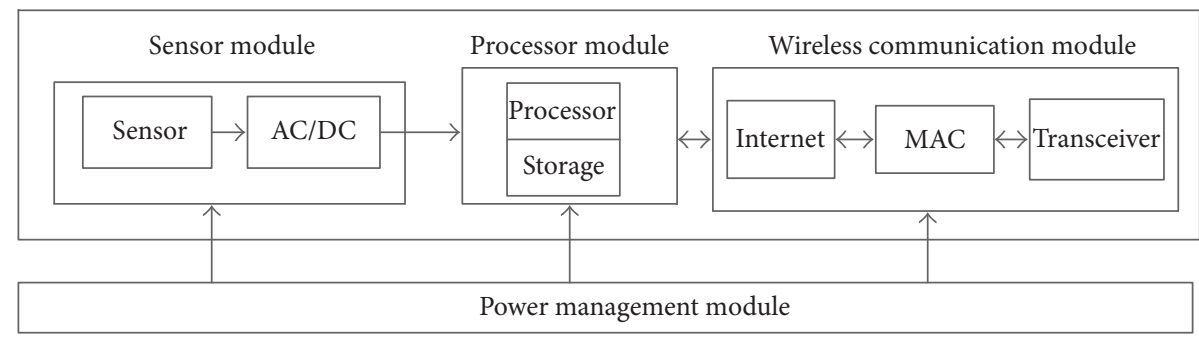

Figure 2: Sensor node architecture.

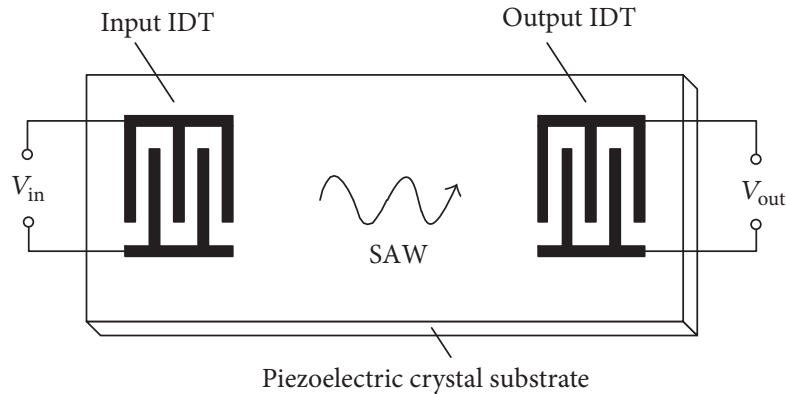

FIGURE 3: Schematic of the double-ended fixed sheet.

SAW sensors have excellent properties, such as passivity, small size, low cost, excellent temperature stability, high reliability, and high reproducibility, whose development based on a micro force sensor has been oriented toward intelligent wireless networks. Therefore, it is highly possible to use SAW pressure sensors in wireless sensor networks.

\section{SAW Based on a Micro Force Sensor}

A SAW sensor is a new type of sensor that combines SAW, thin film, and electronic technology. This sensor translates different physical, chemical, and biomass information around SAW devices into changes in the SAW oscillator frequency by detecting the parameter variation in the frequency to monitor acceleration, temperature, humidity, pressure, shear, and bend. This device also uses the piezoelectric properties and temperature stability of crystals and the frequency signal instead of the conventional pressure sensor with a voltage signal. This approach enhances the signal processing of the device in terms of its digital feature and stable performance. SAW sensor technology is relatively mature at present; researchers have successfully developed pressure, temperature, mass, humidity, and gas sensors.

3.1. Principle of SAW Device. The basic structure of a SAW device is shown in Figure 3. This device manufactures two acoustic electric transducers on the piezoelectric characteristic substrate material-polishing surface, which is defined as the transmitting and receiving IDT (i.e., input and output IDT, resp.). An electrical signal applied on the input IDT generates an opposite polarity potential in two busbars such that an adjacent electric field is generated between two finger pair types. The piezoelectric material surface experiences strain in the electric field, when it affects the substrate with inverse piezoelectric. If the applied electrical signal is alternating, the

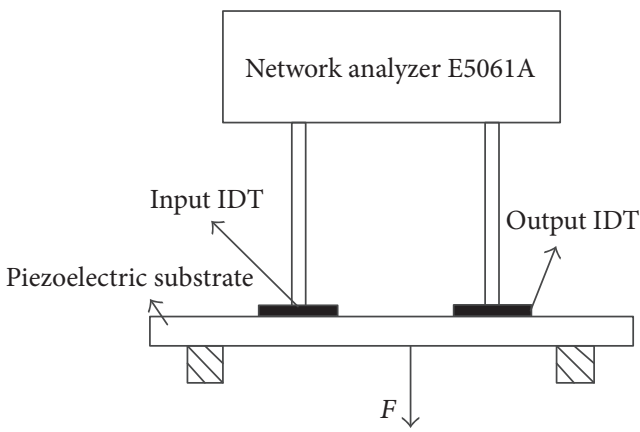

FIGURE 4: Schematic of the SAW-based micro force sensor.

deformation of the piezoelectric material surface propagates through the piezoelectric material surface; this process is called the SAW excitation process. It can produce an electric charge in certain directions of the piezoelectric materials through the output IDT excitation, which the SAW sensor propagates. Given the presence of the interdigital electrode, the charge accumulates on the electrode such that the output IDT busbars can check the corresponding electrical signal [21].

\subsection{Basic Structure and Principle of a SAW Based on a Micro Force Sensor}

3.2.1. Basic Structure of a SAW Based on a Micro Force Sensor. The output frequency of the SAW based on a micro force sensor is conducted using the network analyzer equipment E5061A. Force-measuring elements employ a cantilever beam loaded with a pressure of $0 \mathrm{kPa}$ to $20 \mathrm{kPa}$ as shown in Figure 4.

The piezoelectric substrate adopts a cantilever structure where two ends are fixed and the middle is loaded with micro-pressure. The cantilever experiences deformation when micro-pressure is loaded at the middle of the cantilever. The SAW propagation path is consequently changed. Otherwise, the output frequency is also changed and can detect the micro-pressure loaded on the cantilever.

3.2.2. Principle of Operation. The center frequency of the SAW device is $f$; the equation is as follows:

$$
f=\frac{v}{\lambda}
$$

where $v$ is the propagation velocity of the SAW on the surface of the piezoelectric substrate material and $\lambda$ is the SAW length. Thus

$$
v \approx \sqrt{\frac{E}{\rho}},
$$


where $E$ is the elastic modulus of the piezoelectric substrate material and $\rho$ is the density of the piezoelectric substrate material.

Equation (3) can be derived from (1) and (2) as follows:

$$
f=\frac{\sqrt{E / \rho}}{\lambda} .
$$

Given that the distribution of the IDT fingers is homogeneous, the relationship between the wavelength $\lambda$ and distance $d$ of the IDT fingers is as follows:

$$
\lambda=2 d \text {. }
$$

$v_{0}$ is the propagation velocity of the SAW that does not load the micro-pressure. $\lambda_{0}$, thus, is the SAW center frequency which is written as follows:

$$
f_{0}=\frac{v_{0}}{\lambda_{0}}
$$

The piezoelectric substrate of the cantilever structure experiences deformation when the micro-pressure $F_{m}$ is loaded on two ends of the cantilever. The formula can be written as follows:

$$
\delta=\frac{\Delta d}{d_{0}} .
$$

The relationship between the distance $d$ of the IDT fingers of the SAW device and the piezoelectric substrate deformation $\delta$ is as follows:

$$
d(\delta)=d_{0}+\Delta d=d_{0}+\delta d_{0}=d_{0}(1+\delta) .
$$

The following can be obtained from (4) and (7):

$$
\lambda(\delta)=2 d(\delta)=2 d_{0}(1+\delta)=\lambda_{0}(1+\delta) .
$$

Equation (8) shows that when the micro-pressure is loaded at the middle of the cantilever, the SAW length that propagates along the piezoelectric substrate increases.

Equation (1) shows that the micro-pressure loaded on the cantilever changes the material density $\rho$ and velocity of the SAW pressure sensor. The relationship between $\delta$ and $v$ can be expressed as follows:

$$
v(\delta)=v_{0}(1+K \delta),
$$

where $K$ is a constant of the piezoelectric substrate material.

The function relationship between the output frequency of the SAW based on a micro force sensor and the piezoelectric substrate deformation can be obtained by substituting (8) and (9) in (5):

$$
f(\delta)=\frac{v(\delta)}{\lambda(\delta)}=\frac{v_{0}(1+K \delta)}{\lambda_{0}(1+\delta)} .
$$

The output frequency $\Delta f$ is then equal to the following equation:

$$
\Delta f=f(\delta)-f_{0}=f_{0}\left(\frac{1+K \delta}{1+\delta}-1\right)=f_{0} \frac{\delta(K-1)}{1+\delta} .
$$

Given that the cantilever experiences deformation $\delta \ll 1$ at the micro-pressure $1+\delta \approx 1,(10)$ is rewritten as

$$
\Delta f=f(\delta)-f_{0} \approx f_{0} \delta(K-1) .
$$

The following equation is then obtained:

$$
f(\delta)=\Delta f+f_{0} \approx f_{0}[1+\delta(K-1)]
$$

Equation (13) shows that the sensor output frequency changes the variety of cantilever deformation when the substrate material constant is determined. In other words, using the SAW drift output frequency can measure the micropressure weight loaded on the sensor cantilever.

\section{Creating and Solving the Linear Regression Model of a SAW Based on a Micro Force Sensor}

4.1. Establishing the Model. According to the SAW based on a micro force sensor, input variable (micro-pressure) and output variable (frequency) can be recorded. The difference between the input and output variables is the linear function relationship established in what follows. From (13), the function relationship between the micro-pressure loaded on the sensor $\left(F_{m}\right)$ and the difference frequency $(\Delta f)$ of the output frequency can be written as follows:

$$
\begin{aligned}
F(\Delta f)= & k_{0}+k_{1} \Delta f+k_{2} \Delta f^{2}+k_{3} \Delta f^{3}+\cdots \\
& +k_{n} \Delta f^{n} \cdots
\end{aligned}
$$

The least squares method can be used to solve (14) [22].

Given the experimental data sample $\left(F_{i}, \Delta f_{i} i=1,2, \ldots\right.$, $n$ ) and setting $n=6,(14)$ is equal to

$$
\begin{aligned}
F_{i}= & k_{0}+k_{1} \Delta f_{i}+k_{2} \Delta f_{i}^{2}+k_{3} \Delta f_{i}^{3}+k_{4} \Delta f_{i}^{4}+k_{5} \Delta f_{i}^{5} \\
& +k_{6} \Delta f_{i}^{6},
\end{aligned}
$$

where the regression coefficients $k_{0}, k_{1}, k_{2}, k_{3}, k_{4}, k_{5}$, and $k_{6}$ can be estimated using the least squares.

4.2. Least Squares. A series of data pairs, such as $\left(x_{1}, y_{1}, x_{2}\right.$, $\left.y_{2}, \ldots, x_{m}, y_{m}\right)$, can generally be obtained using the principle of the least squares method and by researching the relationship of the two variables $(x, y)$. If these points are nearly a straight line, we can set the line equation that describes the data in the $x-y$ Cartesian coordinate system as follows:

$$
Y_{j}=a_{0}+a_{1} X
$$

where $a_{0}$ and $a_{1}$ are arbitrary real numbers.

Establishing a linear equation to determine $a_{0}$ and $a_{1}$ is necessary to obtain the sum of squares of the deviations $\sum\left(Y_{i}-Y_{j}\right)^{2}$ of the measured value $Y_{j}$ and the calculated value $Y_{j}$ from (16) as follows:

$$
Q=\sum\left(Y_{i}-Y_{j}\right)^{2}
$$


According to the least squares method, $Q$ is the sum of variance between the predicted dependent variable and its actual value. (17):

The following equation is obtained by substituting (16) in

$$
Q=\sum\left(Y_{i}-a_{0}-a_{1} X_{i}\right)^{2}
$$

The partial derivatives of $a_{0}$ and $a_{1}$ can be calculated using the function $Q$, when $\sum\left(Y_{i}-Y_{j}\right)^{2}$ is at its minimum. The two partial derivatives are set to equal zero:

$$
\begin{aligned}
& \left.\frac{\partial Q\left(a_{0}, a_{1}\right)}{\partial a_{0}}\right|_{a_{0}=\widehat{a_{0}}}=0, \\
& \left.\frac{\partial Q\left(a_{0}, a_{1}\right)}{\partial a_{1}}\right|_{a_{1}=\widehat{a_{1}}}=0 .
\end{aligned}
$$

Thus,

$$
\begin{gathered}
\sum a_{0}+a_{1} \sum X_{i}=\sum Y_{i}, \\
a_{0} \sum X_{i}+a_{1} \sum X_{i}^{2}=\sum X_{i} Y_{i} .
\end{gathered}
$$

The two equations of the unknown $a_{0}$ and $a_{1}$ can be calculated and (16) solved [23].

4.3. Analysis of Solving the Model. According to the least squares method in (16) to (20), the sum of the variances $Q$ between the predicted value $F_{i}$ and the actual value $F$ in (15) is given as

$$
Q\left(k_{0}-k_{6}\right)=\sum_{i=1}^{N} \Delta_{i}{ }^{2}=\sum_{i=1}^{N}\left[F_{i}-F\right]^{2},
$$

where $N$ is the sampling point of the independent variables. The least squares estimation therefore calculates the minimum value of $Q$, which implies that $\widehat{k_{0}}, \widehat{k_{1}}, \widehat{k_{2}}, \widehat{k_{3}}, \widehat{k_{4}}, \widehat{k_{5}}$, and $\widehat{k_{6}}$ must be suitable for the following equation:

$$
\begin{aligned}
& Q\left(\widehat{k_{0}}, \widehat{k_{1}}, \widehat{k_{2}}, \widehat{k_{3}}, \widehat{k_{4}}, \widehat{k_{5}}, \widehat{k_{6}}\right)=\sum_{i=1}^{N}\left[F_{i}-F\right]^{2} \\
& \quad=\underset{\widehat{k_{0}}-\widehat{m}_{i}}{\min _{i=1}^{N}}\left[F_{i}-F\right]^{2}=\underset{\widehat{k_{0}}-\widehat{m i n}_{6}}{ } \sum_{i=1}^{N}\left[k_{0}+k_{1} \Delta f_{i}+k_{2} \Delta f_{i}^{2}\right. \\
& \left.\quad+k_{3} \Delta f_{i}^{3}+k_{4} \Delta f_{i}^{4}+k_{5} \Delta f_{i}^{5}+k_{6} \Delta f_{i}^{6}-F\right]^{2}
\end{aligned}
$$

where $\widehat{k_{0}}, \widehat{k_{1}}, \widehat{k_{2}}, \widehat{k_{3}}, \widehat{k_{4}}, \widehat{k_{5}}$, and $\widehat{k_{6}}$ are the least squares estimations of $k_{0}, k_{1}, k_{2}, k_{3}, k_{4}, k_{5}$, and $k_{6}$, respectively.
Setting the equations

$$
\begin{aligned}
& \left.\frac{\partial Q\left(k_{0}, k_{1}, k_{2}, k_{3}, k_{4}, k_{5}\right)}{\partial k_{0}}\right|_{k_{0}=\widehat{k_{0}}}=0, \\
& \left.\frac{\partial Q\left(k_{0}, k_{1}, k_{2}, k_{3}, k_{4}, k_{5}\right)}{\partial k_{1}}\right|_{k_{0}=\widehat{k_{1}}}=0, \\
& \left.\frac{\partial Q\left(k_{0}, k_{1}, k_{2}, k_{3}, k_{4}, k_{5}\right)}{\partial k_{2}}\right|_{k_{0}=\widehat{k_{2}}}=0, \\
& \left.\frac{\partial Q\left(k_{0}, k_{1}, k_{2}, k_{3}, k_{4}, k_{5}\right)}{\partial k_{3}}\right|_{k_{0}=\widehat{k_{3}}}=0, \\
& \left.\frac{\partial Q\left(k_{0}, k_{1}, k_{2}, k_{3}, k_{4}, k_{5}\right)}{\partial k_{4}}\right|_{k_{0}=\widehat{k_{4}}}=0, \\
& \left.\frac{\partial Q\left(k_{0}, k_{1}, k_{2}, k_{3}, k_{4}, k_{5}\right)}{\partial k_{5}}\right|_{k_{0}=\widehat{k_{5}}}=0, \\
& \left.\frac{\partial Q\left(k_{0}, k_{1}, k_{2}, k_{3}, k_{4}, k_{5}\right)}{\partial k_{6}}\right|_{k_{0}=\widehat{k_{6}}}=0,
\end{aligned}
$$

we can obtain the following:

$$
\begin{aligned}
& \sum_{i=1}^{N}\left[\left(\widehat{k_{0}}+\widehat{k_{1}} \Delta f_{i}+\widehat{k_{2}} \Delta f_{i}^{2}+\widehat{k_{3}} \Delta f_{i}^{3}+\widehat{k_{4}} \Delta f_{i}^{4}+\widehat{k_{5}} \Delta f_{i}^{5}\right.\right. \\
& \left.\left.+\widehat{k_{6}} \Delta f_{i}^{6}\right)-F_{i}\right]=0, \\
& \sum_{i=1}^{N}\left[\left(\widehat{k_{0}}+\widehat{k_{1}} \Delta f_{i}+\widehat{k_{2}} \Delta f_{i}^{2}+\widehat{k_{3}} \Delta f_{i}^{3}+\widehat{k_{4}} \Delta f_{i}^{4}+\widehat{k_{5}} \Delta f_{i}^{5}\right.\right. \\
& \left.\left.+\widehat{k_{6}} \Delta f_{i}^{6}\right)-F_{i}\right] \Delta f_{i}=0, \\
& \sum_{i=1}^{N}\left[\left(\widehat{k_{0}}+\widehat{k_{1}} \Delta f_{i}+\widehat{k_{2}} \Delta f_{i}^{2}+\widehat{k_{3}} \Delta f_{i}^{3}+\widehat{k_{4}} \Delta f_{i}^{4}+\widehat{k_{5}} \Delta f_{i}^{5}\right.\right. \\
& \left.\left.+\widehat{k_{6}} \Delta f_{i}^{6}\right)-F_{i}\right] \Delta f_{i}^{2}=0, \\
& \sum_{i=1}^{N}\left[\left(\widehat{k_{0}}+\widehat{k_{1}} \Delta f_{i}+\widehat{k_{2}} \Delta f_{i}^{2}+\widehat{k_{3}} \Delta f_{i}^{3}+\widehat{k_{4}} \Delta f_{i}^{4}+\widehat{k_{5}} \Delta f_{i}^{5}\right.\right. \\
& \left.\left.+\widehat{k_{6}} \Delta f_{i}^{6}\right)-F_{i}\right] \Delta f_{i}^{3}=0, \\
& \sum_{i=1}^{N}\left[\left(\widehat{k_{0}}+\widehat{k_{1}} \Delta f_{i}+\widehat{k_{2}} \Delta f_{i}^{2}+\widehat{k_{3}} \Delta f_{i}^{3}+\widehat{k_{4}} \Delta f_{i}^{4}+\widehat{k_{5}} \Delta f_{i}^{5}\right.\right. \\
& \left.\left.+\widehat{k_{6}} \Delta f_{i}^{6}\right)-F_{i}\right] \Delta f_{i}^{4}=0, \\
& \sum_{i=1}^{N}\left[\left(\widehat{k_{0}}+\widehat{k_{1}} \Delta f_{i}+\widehat{k_{2}} \Delta f_{i}^{2}+\widehat{k_{3}} \Delta f_{i}^{3}+\widehat{k_{4}} \Delta f_{i}^{4}+\widehat{k_{5}} \Delta f_{i}^{5}\right.\right. \\
& \left.\left.+\widehat{k_{6}} \Delta f_{i}^{6}\right)-F_{i}\right] \Delta f_{i}^{5}=0, \\
& \sum_{i=1}^{N}\left[\left(\widehat{k_{0}}+\widehat{k_{1}} \Delta f_{i}+\widehat{k_{2}} \Delta f_{i}^{2}+\widehat{k_{3}} \Delta f_{i}^{3}+\widehat{k_{4}} \Delta f_{i}^{4}+\widehat{k_{5}} \Delta f_{i}^{5}\right.\right. \\
& \left.\left.+\widehat{k_{6}} \Delta f_{i}^{6}\right)-F_{i}\right] \Delta f_{i}^{6}=0 .
\end{aligned}
$$


Further simplifying the said equations we obtain the following:

$$
\begin{aligned}
& N \widehat{k_{0}}+\widehat{k_{1}} \sum_{i=1}^{N} \Delta f_{i}+\widehat{k_{2}} \sum_{i=1}^{N} \Delta f_{i}^{2}+\widehat{k_{3}} \sum_{i=1}^{N} \Delta f_{i}^{3}+\widehat{k_{4}} \sum_{i=1}^{N} \Delta f_{i}^{4} \\
& +\widehat{k}_{5} \sum_{i=1}^{N} \Delta f_{i}^{5}+\widehat{k}_{6} \sum_{i=1}^{N} \Delta f_{i}^{6}=\sum_{i=1}^{N} F_{i}, \\
& \widehat{k_{0}} \sum_{i=1}^{N} \Delta f_{i}+\widehat{k}_{1} \sum_{i=1}^{N} \Delta f^{2}{ }_{i}+\widehat{k_{2}} \sum_{i=1}^{N} \Delta f_{i}^{3}+\widehat{k_{3}} \sum_{i=1}^{N} \Delta f_{i}^{4} \\
& +\widehat{k}_{4} \sum_{i=1}^{N} \Delta f_{i}^{5}+\widehat{k_{5}} \sum_{i=1}^{N} \Delta f_{i}^{6}+\widehat{k}_{6} \sum_{i=1}^{N} \Delta f_{i}^{7}=\sum_{i=1}^{N} F_{i} \Delta f_{i}, \\
& \widehat{k_{0}} \sum_{i=1}^{N} \Delta f_{i}^{2}+\widehat{k_{1}} \sum_{i=1}^{N} \Delta f_{i}^{3}+\widehat{k}_{2} \sum_{i=1}^{N} \Delta f_{i}^{4}+\widehat{k}_{3} \sum_{i=1}^{N} \Delta f_{i}^{5} \\
& +\widehat{k}_{4} \sum_{i=1}^{N} \Delta f_{i}^{6}+\widehat{k}_{5} \sum_{i=1}^{N} \Delta f_{i}^{7}+\widehat{k}_{6} \sum_{i=1}^{N} \Delta f_{i}^{8}=\sum_{i=1}^{N} F_{i} \Delta f_{i}^{2}, \\
& \widehat{k_{0}} \sum_{i=1}^{N} \Delta f_{i}^{3}+\widehat{k_{1}} \sum_{i=1}^{N} \Delta f^{4}{ }_{i}+\widehat{k_{2}} \sum_{i=1}^{N} \Delta f_{i}^{5}+\widehat{k_{3}} \sum_{i=1}^{N} \Delta f_{i}^{6} \\
& +\widehat{k_{4}} \sum_{i=1}^{N} \Delta f_{i}^{7}+\widehat{k}_{5} \sum_{i=1}^{N} \Delta f_{i}^{8}+\widehat{k}_{6} \sum_{i=1}^{N} \Delta f_{i}^{9}=\sum_{i=1}^{N} F_{i} \Delta f_{i}^{3}, \\
& \widehat{k_{0}} \sum_{i=1}^{N} \Delta f_{i}^{4}+\widehat{k_{1}} \sum_{i=1}^{N} \Delta f_{i}^{5}+\widehat{k_{2}} \sum_{i=1}^{N} \Delta f_{i}^{6}+\widehat{k_{3}} \sum_{i=1}^{N} \Delta f_{i}^{7} \\
& +\widehat{k_{4}} \sum_{i=1}^{N} \Delta f_{i}^{8}+\widehat{k}_{5} \sum_{i=1}^{N} \Delta f_{i}^{9}+\widehat{k_{6}} \sum_{i=1}^{N} \Delta f_{i}^{10}=\sum_{i=1}^{N} F_{i} \Delta f_{i}^{4}, \\
& \widehat{k_{0}} \sum_{i=1}^{N} \Delta f_{i}^{5}+\widehat{k_{1}} \sum_{i=1}^{N} \Delta f_{i}^{6}+\widehat{k_{2}} \sum_{i=1}^{N} \Delta f_{i}^{7}+\widehat{k}_{3} \sum_{i=1}^{N} \Delta f_{i}^{8} \\
& +\widehat{k_{4}} \sum_{i=1}^{N} \Delta f_{i}^{9}+\widehat{k_{5}} \sum_{i=1}^{N} \Delta f_{i}^{10}+\widehat{k_{6}} \sum_{i=1}^{N} \Delta f_{i}^{11}=\sum_{i=1}^{N} F_{i} \Delta f_{i}^{5}, \\
& \widehat{k_{0}} \sum_{i=1}^{N} \Delta f_{i}^{6}+\widehat{k}_{1} \sum_{i=1}^{N} \Delta f_{i}^{7}+\widehat{k}_{2} \sum_{i=1}^{N} \Delta f_{i}^{8}+\widehat{k}_{3} \sum_{i=1}^{N} \Delta f_{i}^{9} \\
& +\widehat{k_{4}} \sum_{i=1}^{N} \Delta f_{i}^{10}+\widehat{k_{5}} \sum_{i=1}^{N} \Delta f_{i}^{11} \\
& +\widehat{k_{6}} \sum_{i=1}^{N} \Delta f_{i}^{12}=\sum_{i=1}^{N} F_{i} \Delta f_{i}^{6} .
\end{aligned}
$$

The following equations are set:

$$
\begin{aligned}
& A=\sum_{i=1}^{N} \Delta f_{i}, \\
& B=\sum_{i=1}^{N} \Delta f^{2}, \\
& C=\sum_{i=1}^{N} \Delta f_{i}^{3}, \\
& D=\sum_{i=1}^{N} \Delta f_{i}^{4},
\end{aligned}
$$

$$
\begin{aligned}
E & =\sum_{i=1}^{N} \Delta f_{i}^{5}, \\
F & =\sum_{i=1}^{N} \Delta f_{i}^{6}, \\
G & =\sum_{i=1}^{N} \Delta f_{i}^{7}, \\
H & =\sum_{i=1}^{N} \Delta f_{i}^{8}, \\
I & =\sum_{i=1}^{N} \Delta f_{i}^{9}, \\
J & =\sum_{i=1}^{N} \Delta f_{i}^{10}, \\
K & =\sum_{i=1}^{N} \Delta f_{i}^{11}, \\
L & =\sum_{i=1}^{N} \Delta f_{i}^{12}, \\
M & =\sum_{i=1}^{N} F_{i} \Delta f_{i}^{5}, \\
M & =\sum_{i=1}^{N} F_{i}, \\
P & =\sum_{i=1}^{N} F_{i} \Delta f_{i}, \\
S & =\sum_{i=1}^{N} F_{i} \Delta f_{i}^{2}, \\
& =\sum_{i=1}^{N} F_{i} \Delta f_{i}^{3} \\
\hline & F_{i} \Delta f_{i}^{4},
\end{aligned}
$$

Equation (25) can be written as follows:

$$
\begin{aligned}
& N \widehat{k_{0}}+A \widehat{k_{1}}+B \widehat{k_{2}}+C \widehat{k_{3}}+D \widehat{k_{4}}+E \widehat{k_{5}}+F \widehat{k_{6}}=M, \\
& A \widehat{k_{0}}+B \widehat{k_{1}}+C \widehat{k_{2}}+D \widehat{k_{3}}+E \widehat{k_{4}}+F \widehat{k_{5}}+G \widehat{k_{6}}=P, \\
& B \widehat{k_{0}}+C \widehat{k_{1}}+D \widehat{k_{2}}+E \widehat{k_{3}}+F \widehat{k_{4}}+G \widehat{k_{5}}+H \widehat{k_{6}}=Q, \\
& C \widehat{k_{0}}+D \widehat{k_{1}}+E \widehat{k_{2}}+F \widehat{k_{3}}+G \widehat{k_{4}}+H \widehat{k_{5}}+I \widehat{k_{6}}=R, \\
& D \widehat{k_{0}}+E \widehat{k_{1}}+F \widehat{k_{2}}+G \widehat{k_{3}}+H \widehat{k_{4}}+I \widehat{k_{5}}+J \widehat{k_{6}}=S,
\end{aligned}
$$


TABLE 1: Frequency and pressure experiment data.

\begin{tabular}{lccc}
\hline$F_{m}(\mathrm{kPa})$ & $f(\mathrm{MHz})$ & $f(\mathrm{MHz})$ & $\Delta f(\mathrm{~Hz})$ \\
\hline 0 & 40.784663 & 40.784744 & 81 \\
1 & 40.788365 & 40.788453 & 88 \\
2 & 40.787996 & 40.788086 & 90 \\
3 & 40.788788 & 40.788881 & 93 \\
4 & 40.790232 & 40.790326 & 94 \\
5 & 40.789643 & 40.789746 & 103 \\
6 & 40.788437 & 40.788542 & 105 \\
7 & 40.790993 & 40.791100 & 107 \\
8 & 40.788762 & 40.788871 & 109 \\
9 & 40.790272 & 40.790385 & 113 \\
10 & 40.790215 & 40.790331 & 116 \\
11 & 40.788104 & 40.788221 & 117 \\
12 & 40.788600 & 40.788721 & 121 \\
13 & 40.789033 & 40.789155 & 122 \\
14 & 40.788988 & 40.789112 & 124 \\
15 & 40.787255 & 40.787383 & 128 \\
16 & 40.788580 & 40.788712 & 132 \\
17 & 40.784916 & 40.785051 & 135 \\
18 & 40.786062 & 40.786201 & 139 \\
19 & 40.786068 & 40.786211 & 143 \\
20 & 40.785977 & 40.786124 & 147 \\
\hline
\end{tabular}

$$
\begin{aligned}
& E \widehat{k_{0}}+F \widehat{k_{1}}+G \widehat{k_{2}}+H \widehat{k_{3}}+I \widehat{k_{4}}+J \widehat{k_{5}}+K \widehat{k_{6}}=T, \\
& F \widehat{k_{0}}+G \widehat{k_{1}}+H \widehat{k_{2}}+I \widehat{k_{3}}+J \widehat{k}_{4}+K \widehat{k_{5}}+L \widehat{k_{6}}=U .
\end{aligned}
$$

Solving (27) yields the estimated value $\widehat{k_{0}}-\widehat{k_{6}}$ of the parameters $k_{0}-k_{6}$ of the regression analysis model shown in (15). The input and output variable regression of the SAW based on a micro force sensor can be calculated by solving (15).

\subsection{Model Solution}

4.4.1. Experimental Setup. On the basis of previous study, different size sensors were fabricated. Then, the network analyzer E5061A is used for testing (Figure 6).

Force-measuring elements employ a cantilever beam loaded with a pressure of $0 \mathrm{kPa}$ to $20 \mathrm{kPa}$; a pressure of $1 \mathrm{kPa}$ is added to the beam each time. Because of the fluctuation of the frequency in the measurement, it is necessary to read the data after a period of time, and the more the data is, the more accurate the frequency conversion formula will be.

Environment of equipment is not complicated; the test can be operated on the experimental platform. And there is no special requirement for operators; they need to operate carefully.

4.4.2. Experimental Data. The experimental data were measured in the laboratory as shown in Table 1 [24]. $F_{m}(\mathrm{kPa})$ is the acting force on the piezoelectric substrate, $f$ is the minimum and maximum frequencies tested by the network analyzer, and $\Delta f$ is the difference between the maximum and minimum frequencies.
4.4.3. Selecting the Degree of Polynomial Fitting. This study uses six-degree polynomial fitting, which can be proven directly by the polynomial fitting procedure three to seven times. The main program is as follows:

$$
\begin{aligned}
& x=[81,88,90,93,94,103,105,107,109,113,116,117,121, \\
& 122,124,128,132,135,139,143,147] ; \\
& y=[0,1,2,3,4,5,6,7,8,9,10,11,12,13,14,15,16,17, \\
& 18,19,20] ; \\
& P=\text { zeros }(5,7) ; \% \text { Define the matrix size } \\
& \text { for } k=2: 6 ; \% 3 \text { to } 7 \text { times fitting } \\
& {[P t, S]=\text { polyfit }(x, y, k) ; \% \text { Polynomial fitting }} \\
& P(k-1,1: k+1)=P t ; \% \text { Assign } P t \text { to matrix } P \\
& Y=\text { polyval }(P t, x, S) ; \% \text { Calculate the fitting values of } \\
& x \\
& D t(k-1)=\operatorname{std}(Y-y) ; \% \text { Calculate the standard devia- } \\
& \text { tion between the fitting value and original data } \\
& \text { end } \\
& \text { Results are as follows: } \\
& \text { Columns } 1 \text { to } 5 \\
& 0.576165303485416 \\
& 0.405216710673834 \\
& 0.393676218911461 \\
& 0.364152373789325 \\
& 0.347679434090903 .
\end{aligned}
$$

The variance decreases rapidly when the polynomial number is more than 5 . The increase in the number also quickly decreases the errors. Thus, a six-degree polynomial fits the laboratory data.

4.4.4. Solving the Polyfit Function. This study constructs an analytic function based on the principle of curve fitting, which is the polynomial $y=a_{1} x^{n}+a_{2} x^{n-1}+\cdots+a_{n} x+a_{n+1}$. This function ensures that the original discrete points on the data set are as close as possible to the given value. After solving the function, the following fitted values are obtained:

$$
\begin{aligned}
p= & 4.192893268467821 e-09, \\
& -2.793471478615071 e \\
& -06,7.674258962870516 e-04, \\
& -0.111309364487564,8.994744948450235, \\
& -3.839297860714657 e \\
& +02,6.758923327690160 e+03, \\
y & =[0.00000000419289326846782108, \\
& -0.00000279347147861507054, \\
& 0.000767425896287051595, \\
& -0.111309364487563539,8.99474494845023465, \\
& -383.929786071465742,6758.92332769015957] .
\end{aligned}
$$


$\%$ in the form of diminishing a dimension provides the fitting polynomial coefficients.

4.4.5. Matrix Calculation. Solving (16) requires the calculation of an approach to a computed matrix in MATLAB. The obtained results are given polynomial coefficients in the form of an increasing dimension number.

The value of $\widehat{k_{0}}-\widehat{k_{6}}$ is as follows:

$$
\begin{aligned}
x 1 & =6633.023822460154, \\
& -384.0079835318029,8.905647996212356, \\
& -0.114164102269569,0.000710263767188689, \\
& -0.000002698678654041,0.0000000040823517085 .
\end{aligned}
$$
follows:

The results of $y$ that retain 18 significant figures are as

$$
\begin{aligned}
y= & 6.633023822460154 e+3, \\
& -3.840079835318029 e \\
& +2,8.905647996212356 \\
& -0.114164102269569,0.710263767188689 e \\
& -3,-0.2698678654041 e-5,0.40823517085 e \\
& -8 .
\end{aligned}
$$

\section{Fitting Results and Curve}

The following arithmetic formula is obtained using the matrix method to solve the final frequency pressure polynomial:

$$
\begin{aligned}
F(\Delta f)= & 6.6330 \times 10^{3}-3.8401 \times 10^{2} \Delta f_{i} \\
& +8.9056 \Delta f_{i}^{2}-0.1142 \Delta f_{i}^{3}+0.7102 \\
& \times 10^{-3} \Delta f_{i}^{4}-0.2698 \times 10^{-5} \Delta f_{i}^{5}+0.4082 \\
& \times 10^{-8} \Delta f_{i}^{6}
\end{aligned}
$$

The polyfit function method can be used to solve the same equation:

$$
\begin{aligned}
F(\Delta f)= & 6.7589 \times 10^{3}-3.8392 \times 10^{2} \Delta f_{i} \\
& +8.9947 \Delta f_{i}^{2}-0.1113 \Delta f_{i}^{3}+0.7674 \\
& \times 10^{-3} \Delta f_{i}^{4}-0.2793 \times 10^{-5} \Delta f_{i}^{5}+0.4193 \\
& \times 10^{-8} \Delta f_{i}^{6}
\end{aligned}
$$

The polyfit function is a curve fitting function that uses the least square parameter in MATLAB. Its principle is to construct an analytic function or polynomial through the discrete points on the data set and ensure that the original discrete points approach the infinity of the given value. The matrix function uses the matrix arithmetic method to solve a data set, which belongs to the method of linear algebra.
Using two methods to calculate the frequency and pressure conversion algorithm results in a generally consistent trend in this study with a certain range of error.

In the picture, the data are as follows:
(1) $(0,81)$
(2) $(1,88)$
(3) $(2,90)$
(4) $(3,93)$
(5) $(4,94)$
(6) $(5,103)$
(7) $(6,105)$
(8) $(7,107)$
(9) $(8,109)$
(10) $(9,113)$
(11) $(10,116)$
(12) $(11,117)$
(13) $(12,121)$
(14) $(13,122)$
(15) $(14,124)$
(16) $(15,128)$
(17) $(16,132)$
(18) $(17,135)$
(19) $(18,139)$
(20) $(19,143)$
(21) $(20,147)$.

Figure 7 shows the polynomial simulating curve. The small red circle shows the relationship between the experimental values of the frequency difference and the experimental values of the pressure. The black line shows the relationship between the experimental values of the frequency difference and the pressure fitting values. The polyfit function also exhibits better polynomial fitting performance than the matrix calculation when the experiment data are significantly large. Unlike other published approaches, the small circle distribution is successfully simulated through the polynomial curve fitting. The normal interfacial stress sensor cannot measure a stress range less than $11 \mathrm{kPa}$. We designed the SAW based on a micro force sensor, which is based on the polyfit function and matrix calculation to overcome precisely this shortcoming. The sensor has better anti-interference and higher accuracy than others, can measure a range of $0 \mathrm{kPa}$ to $20 \mathrm{kPa}$ of microstress, and has good linearity (Figure 5).

\section{Temperature Compensation}

In this paper, a new type of SAW sensors is based on $\mathrm{LiNbO}_{3}$. In order to ensure accuracy of data in the measurement, it is necessary to analyze the temperature compensation. The same reference device and the original measuring device are arranged in parallel. The output difference frequency method 


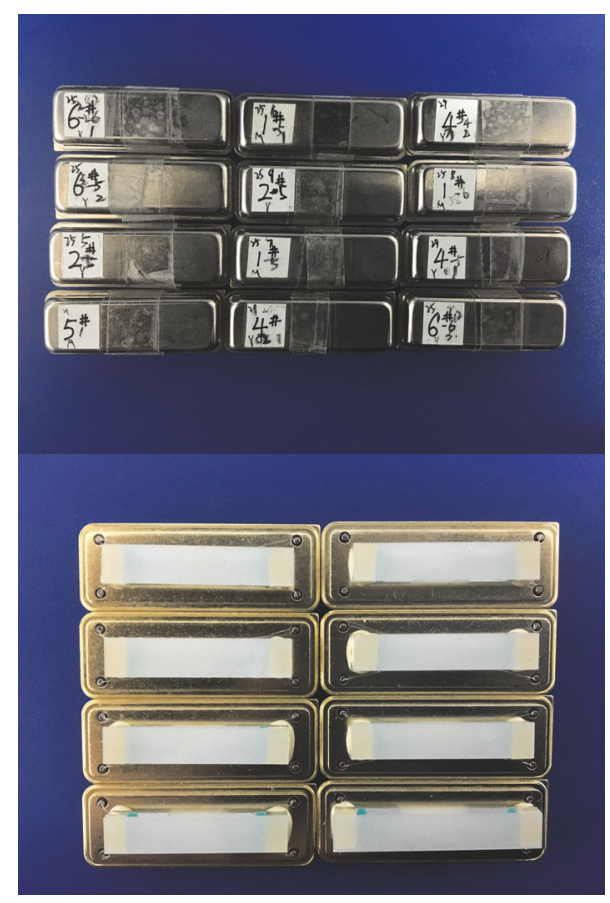

FIgURE 5: Physical maps of SAW based on a micro sensor.
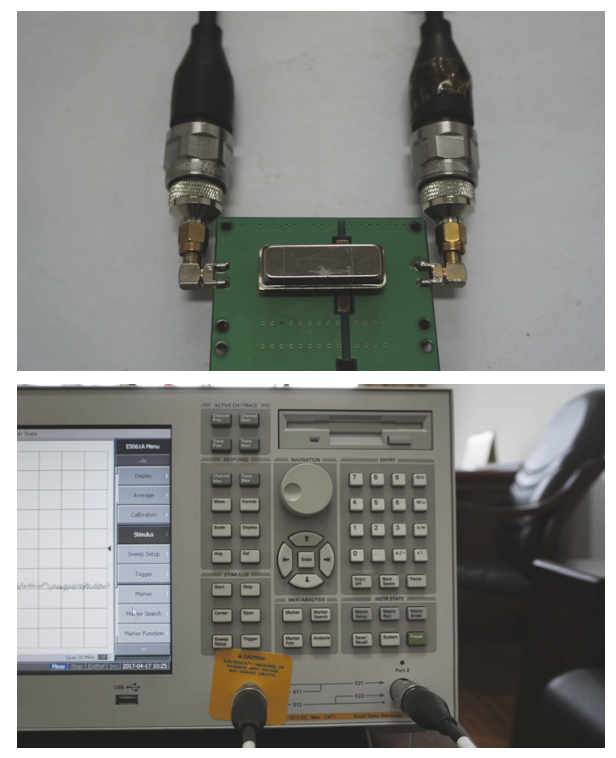

FIGURE 6: The connection of a sensor and the network analyzer.

is adopted to eliminate the interference of temperature, humidity, and noise, as shown in Figure 8.

The output frequency of a SAW sensor is $F_{1}, T_{1}$ is the initial temperature, $T_{2}$ is the ambient temperature, $\Delta T$ is the difference temperature between the initial temperature and ambient temperature, and $\beta_{1}$ is the temperature coefficient of a SAW sensor. $\Delta F$ is the difference output frequency between one sensor and the reference sensor; then (33) is given as

$$
F_{1}=F_{01}+\Delta F+\beta_{1}\left(T_{2}-T_{1}\right)=F_{01}+\Delta F+\beta_{1} \Delta T
$$

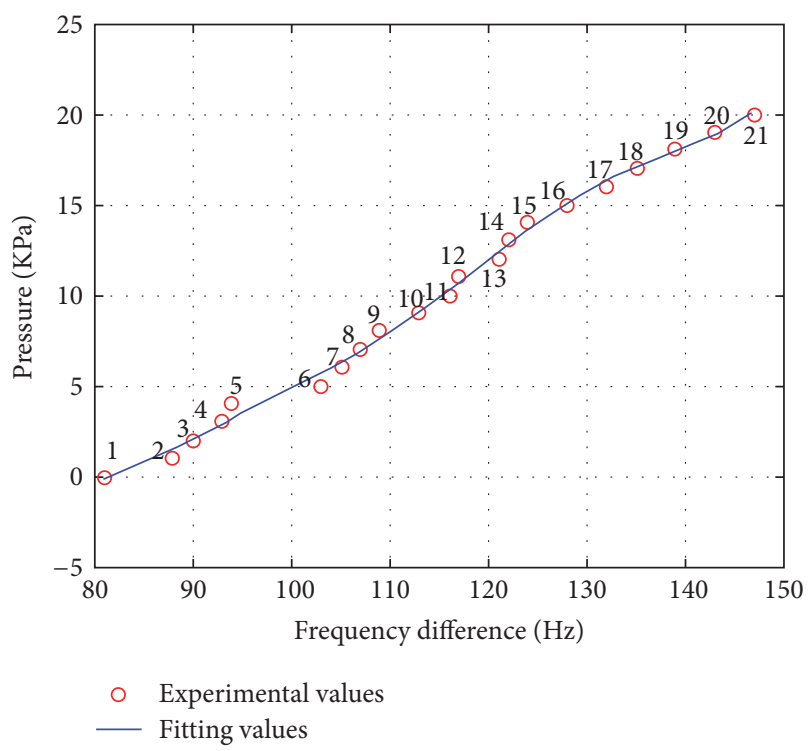

FIGURE 7: Fitting curve of the frequency difference and pressure of a SAW based on a micro force sensor.

where $F_{01}$ is the initial frequency of the output of the sensing device without loading micro-pressure in temperature $T_{1}$. The output frequency of the reference SAW sensor is $F_{2}$; the formula can be written as follows:

$$
F_{2}=F_{02}+\beta_{2} \Delta T,
$$

where $F_{02}$ is the initial frequency of the output of the reference device without loading micro-pressure in temperature $T_{2}$ and $\beta_{2}$ the temperature coefficient of a reference SAW sensor.

The difference frequency $(\Delta F)$ between $F_{1}$ and $F_{2}$ can be calculated:

$$
\Delta F=F_{1}-F_{2}=F_{01}-F_{02}+\Delta F+\left(\beta_{1}-\beta_{2}\right) \Delta T .
$$

When $\beta_{1}$ and $\beta_{2}$ are equal, the difference of the output frequency of sensors is only related to the difference of temperature between the initial temperature and ambient temperature $\Delta T$; then the reference device can completely compensate the influence of temperature on the sensing device.

Due to the difference of SAW devices, temperature coefficients cannot be equal. However, the sensor and the reference sensor have the same material, design, and fabrication; a certain amount of error will not affect the system so that the temperature compensation of SAW sensors can be fully realized.

\section{Conclusion}

WSNs have an active role in the detection of seismic and electromagnetic parameters, temperature, humidity, noise, light intensity, pressure, soil composition, size of a moving object, and speed and direction of the surrounding environment. As a new sensor type, the SAW based on a micro force sensor has high precision, small size, and high reliability 


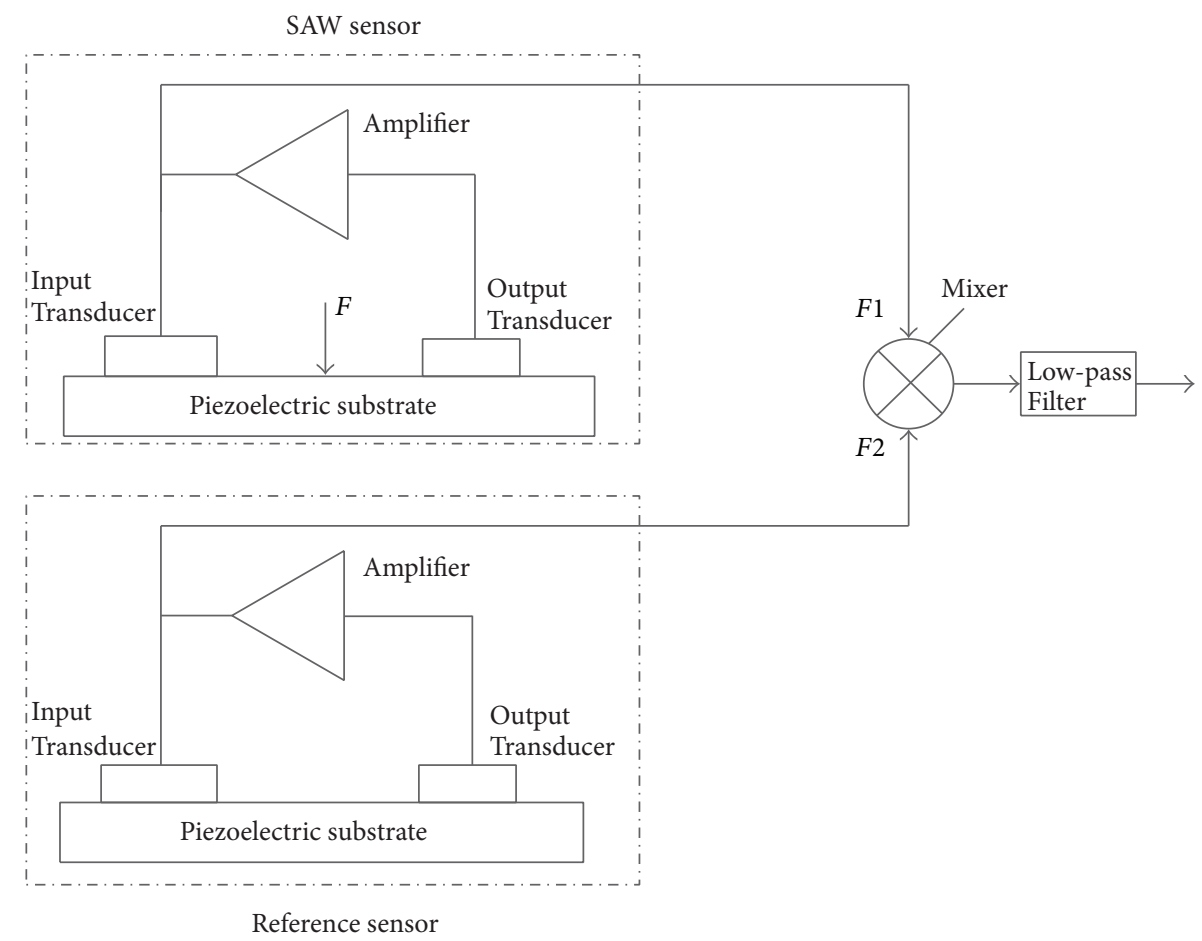

FIGURE 8: Principles of temperature compensation for SAW sensors.

characteristics in line with the high demand for sensors in the rapid development of WSNs.

This paper presents two different methods of analyzing the frequency and pressure conversion optimization algorithm of a SAW based on a micro force sensor. The polyfit function and matrix calculation, which are based on WSNs, SAW detection, and least squares method, have practical applications in the data acquisition of the proposed sensor in WSN nodes.

The study also proposes the pressure difference and frequency conversion method based on the relationship between the frequency and actual pressure data. The difference in the frequency data for different pressure values can also be established through the least squares model. The best estimate for the value of the polynomial coefficients and optimized model can be obtained after determining the minimum sum of the squares of the difference between the predicted and actual values. This approach decreases the measurement error and improves the advantages and competitiveness of the SAW-based micro force sensor in WSNs. SAW sensors in different WSN nodes use the piezoelectric properties and temperature stability of the crystal and the frequency signal instead of the conventional pressure sensor with a voltage signal. This approach enhances the signal processing of the device in terms of its digital feature and stable performance. Nevertheless, several fundamental problems still need to be solved, such as using more than one polynomial function in the fitting curve, expanding the scope of the pressure, and increasing the sampling data. Further studies on these issues should be undertaken in the future.

\section{Conflicts of Interest}

The authors declare that they have no conflicts of interest.

\section{Acknowledgments}

Authors' deepest gratitude goes first and foremost to Professor Chuangchun Zhu, and the authors acknowledge the National Natural Science Foundation of China (no. 61274078), National Basic Research Program of China Textile Industry (no. J201608), and Research and Innovation Project of Graduate Students of Shanghai University of Engineering Science (E3-0903-16-01182) for the financial support and technical assistance.

\section{References}

[1] J. V. Nickerson and S. Olariu, "Protecting with sensor networks: attention and response," in Proceedings of the 40th Annual Hawaii International Conference on System Sciences (HICSS '07), January 2007.

[2] A. Kishtwal, J. Singh, and R. Bhatt, "A review: wireless sensor networks (WSN) and security aspects," International Journal of Engineering Research \& Technology, vol. 3, no. 1, pp. 223-228, 2014.

[3] B. Tang, Q. Huang, L. Deng, and Z. Liu, "Research progress and challenges of wireless sensor networks for machinery equipment condition monitoring," Journal of Vibration, Measurement and Diagnosis, vol. 34, no. 1, pp. 1-7, 2014.

[4] T. Cui, L. Chen, and T. Ho, "Energy efficient opportunistic network coding for wireless networks," in Proceedings of the 27th IEEE Communications Society Conference on Computer Communications (INFOCOM '08), pp. 361-365, April 2008. 
[5] V. Ugrinovskii, "Conditions for detectability in distributed consensus-based observer networks," IEEE Transactions on Automatic Control, vol. 58, no. 10, pp. 2659-2664, 2013.

[6] H. Dong, Z. Wang, and H. Gao, "Distributed H-infinity filtering for a class of markovian jump nonlinear time-delay systems over lossy sensor networks " IEEE Transactions on Industrial Electronics, vol. 60, no. 10, pp. 4665-4672, 1999.

[7] D. Liao and K. Sarabandi, "Optimization of low-profile antennas for applications in unattended ground sensor networks," in Proceedings of the IEEE Antennas and Propagation Society International Symposium, pp. 783-786, Albuquerque, NM, USA, July 2006.

[8] J. Tokyo, "The future of technology," Global Electronics China, vol. 1, no. 5, pp. 4-500, 2007.

[9] J. Su, H. Qiao, Z. Ou, and Y. Zhang, "Sensor-less insertion strategy for an eccentric peg in a hole of the crankshaft and bearing assembly," Assembly Automation, vol. 32, no. 1, pp. 8699, 2012.

[10] Q. Li, B. Shen, Y. Liu, and T. Huang, "Event-triggered Ho state estimation for discrete-time neural networks with mixed time delays and sensor saturations," Neural Computing and Applications, pp. 1-11, 2016.

[11] C. Y. Xiong, J. W. Tian, and J. Liu, "A note on 'Flipping structure: an efficient VLSI architecture for lifting-based discrete wavelet transform," IEEE Transactions on Signal Processing, vol. 54, no. 5, pp. 1910-1916, 2006.

[12] X. Tian, L. Wu, Y.-H. Tan, and J.-W. Tian, "Efficient multiinput/multi-output VLSI architecture for two-dimensional lifting-based discrete wavelet transform," IEEE Transactions on Computers, vol. 60, no. 8, pp. 1207-1211, 2011.

[13] W. Lu, L. Kuang, X. Lü, C. Zhu, T. Zhang, and J. Zhang, "Solution of some problems of single-scale wavelet transform processor using a magnetostatic surface wave device," Metrology and Measurement Systems, vol. 19, no. 4, pp. 685-692, 2012.

[14] D. S. Ballantine, R. M. White, S. J. Martin et al., Acoustic Wave Sensor: Theory, Design, and Physic-Chemical Applications, Academic Press, 1997.

[15] L. Xu, Y. Liu, and X. Fu, "Effects of the van der waals force on the dynamics performance for a micro resonant pressure sensor," Shock and Vibration, vol. 2016, Article ID 3426196, 11 pages, 2016.

[16] W. K. Lu, C. C. Zhu, J. H. Liu, and Q. Liu, "Implementing wavelet transform with SAW elements," Science in China, Series E: Technological Sciences, vol. 46, no. 6, pp. 627-638, 2003.

[17] X. Z. Lü, Interfacial Stress Sensor for Artificial Skin Application, Donghua University, 2012.

[18] M. Jungwirth, H. Scherr, and R. Weigel, "Micromechanical precision pressure sensor incorporating SAW delay lines," Acta Mechanica, vol. 158, no. 3-4, pp. 227-252, 2002.

[19] S. Muntwyler, F. Beyeler, and B. J. Nelson, “Three-axis microforce sensor with sub-micro-Newton measurement uncertainty and tunable force range," Journal of Micromechanics and Microengineering, vol. 20, no. 2, Article ID 025011, pp. 3165-3170, 2010.

[20] Z. Cheng, Research on Wireless Sensor Network Node Based on CC2431, University of Science and Technology of China, Hefei, China, 2009 (Chinese).

[21] A. Binder, G. Bruckner, N. Schobernig, and D. Schmitt, "Wireless surface acoustic wave pressure and temperature sensor with unique identification based on LiNbO3," IEEE Sensors Journal, vol. 13, no. 5, pp. 1801-1805, 2013.
[22] W. K. Lu, C. C. Zhu, J. F. Zhang, C. Shi, and X. Z. Lü, "Study of small size wavelet transform processor and wavelet inversetransform processor using SAW devices," Measurement, vol. 44, no. 5, pp. 994-999, 2011.

[23] K. Z. Zhou, Least-Square Method, Longmen Joint Press, 1951 (Chinese).

[24] Y. Y. Li, W. K. Lu, C. C. Zhu et al., "Acoustic electric generation for Morlet wavelet transform of surface acoustic wave device," Research Journal of Applied Sciences, Engineering and Technology, vol. 5, no. 4, pp. 1203-1207, 2013. 


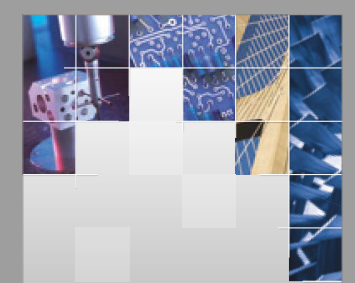

\section{Enfincering}
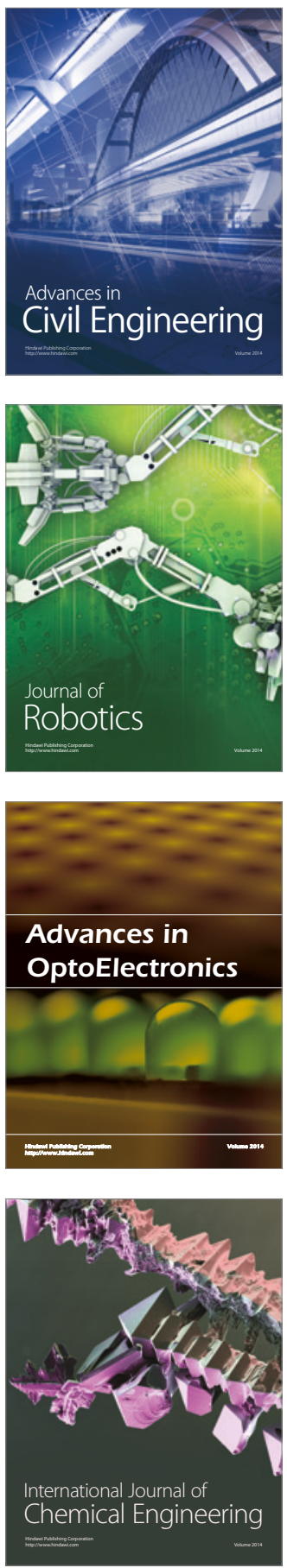

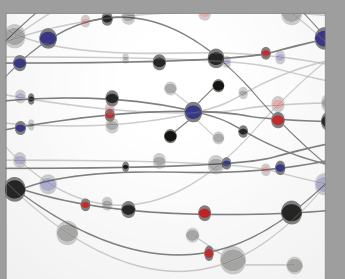

The Scientific World Journal

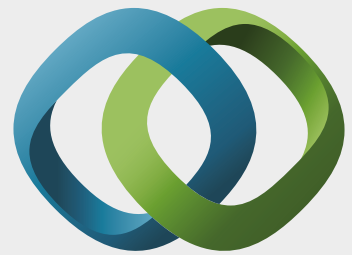

\section{Hindawi}

Submit your manuscripts at

https://www.hindawi.com
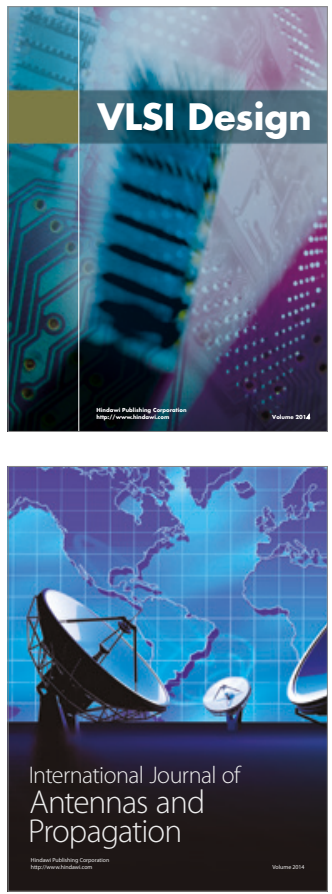

\section{Rotating}

Machinery
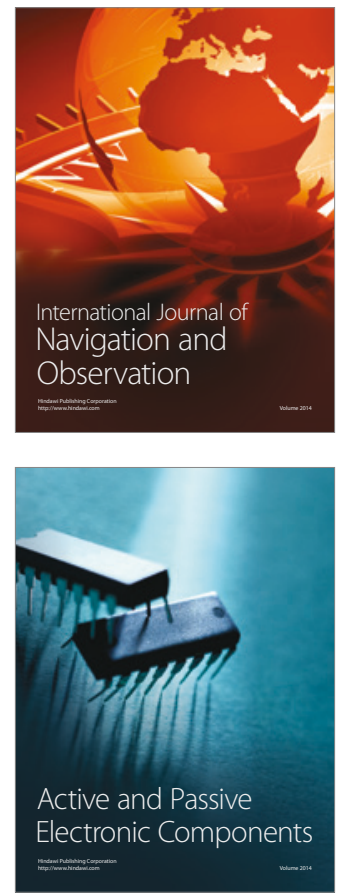
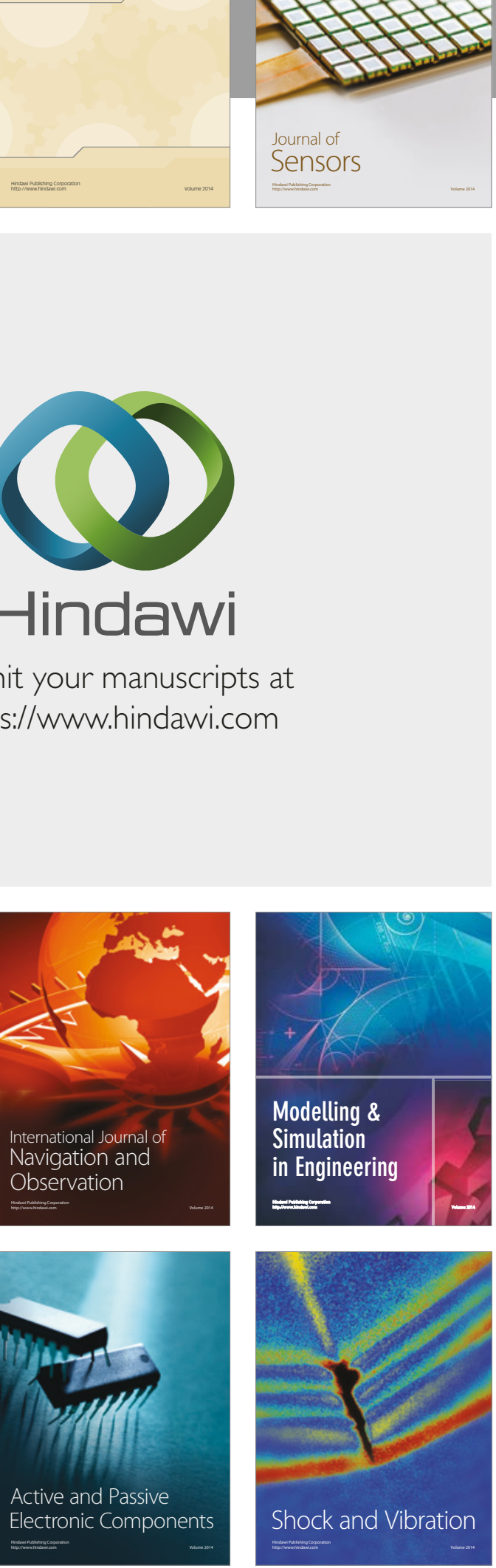
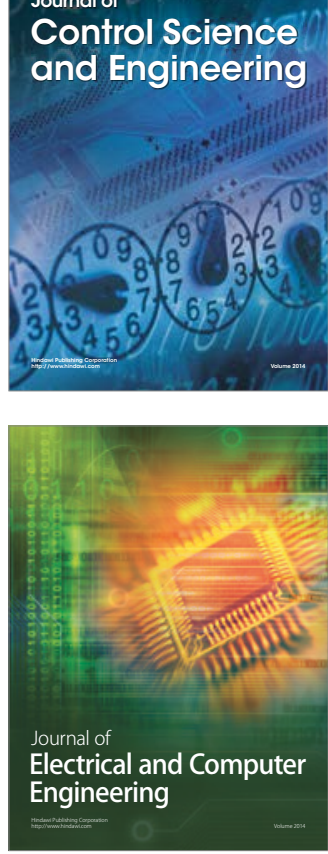

Distributed

Journal of

Control Science

and Engineering
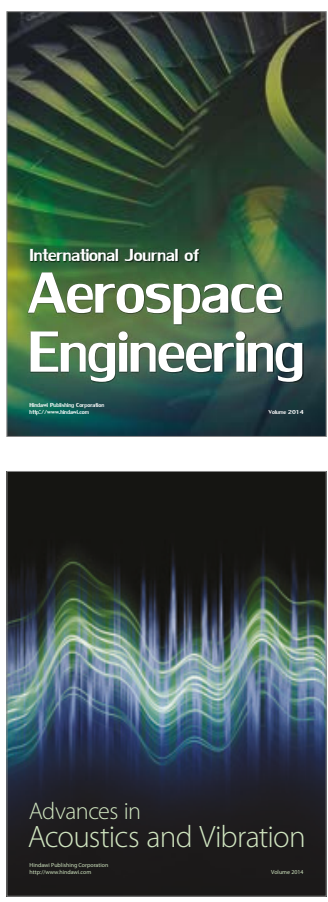

Sensor Networks 